\title{
Existence and asymptotic properties of singular solutions of nonlinear elliptic equations in $R^{n} \backslash\{0\}$
}

Imed Bachar ${ }^{\text {* }}$ (D) and Entesar Aljarallah 2,1

\section{*Correspondence:}

abachar@ksu.edu.sa

${ }^{1}$ College of Science, Mathematics

Department, King Saud University,

P.O. Box 2455, 11451 Riyadh, Saudi

Arabia

Full list of author information is

available at the end of the article

$$
\begin{aligned}
& \text { Abstract } \\
& \text { We consider the following singular semilinear problem } \\
& \qquad\left\{\begin{array}{l}
\Delta u(x)+p(x) u^{\gamma}=0, \quad x \in D \text { (in the distributional sense), } \\
u>0, \text { in } D, \\
\lim _{|x| \rightarrow 0}|x|^{n-2} u(x)=0, \\
\lim _{|x| \rightarrow \infty} u(x)=0,
\end{array}\right.
\end{aligned}
$$

where $\gamma<1, D=\mathbb{R}^{n} \backslash\{0\}(n \geq 3)$ and $p$ is a positive continuous function in $D$, which may be singular at $x=0$. Under sufficient conditions for the weighted function $p(x)$, we prove the existence of a positive continuous solution on $D$, which could blow-up at the origin. The global asymptotic behavior of this solution is also obtained.

MSC: 34B16; 34B18; 34B27

Keywords: Slowly varying functions; Kato class; Asymptotic properties; Blow-up

\section{Introduction and the main result}

Semilinear elliptic partial differential equations of the type

$$
\Delta u(x)+p(x) u^{\gamma}=0
$$

will be considered in $D=\mathbb{R}^{n} \backslash\{0\}(n \geq 3)$, where $\gamma<1$ and $p$ is a positive continuous function in $D$, which may be singular at $x=0$. Our main goal is to establish sufficient conditions for the existence of a positive continuous solution $u(x)$ of (1.1) with specified asymptotic behavior as $|x| \rightarrow 0$ and as $|x| \rightarrow \infty$. Global asymptotic behavior of this solution is also obtained.

The importance of this type of equation in mathematics and applied mathematics has been widely recognized; see, for example, [11-13].

The above equation, subjected to homogeneous Dirichlet boundary conditions, has been intensively studied in the case where $D=\mathbb{R}^{n}(n \geq 3)$. In this sense, the existence

(c) The Author(s) 2022. This article is licensed under a Creative Commons Attribution 4.0 International License, which permits use, sharing, adaptation, distribution and reproduction in any medium or format, as long as you give appropriate credit to the original author(s) and the source, provide a link to the Creative Commons licence, and indicate if changes were made. The images or other third party material in this article are included in the article's Creative Commons licence, unless indicated otherwise in a credit line to the material. If material is not included in the article's Creative Commons licence and your intended use is not permitted by statutory regulation or exceeds the permitted use, you will need to obtain permission directly from the copyright holder. To view a copy of this licence, visit http://creativecommons.org/licenses/by/4.0/. 
of entire positive solutions for any $\gamma<0$, that is the singular case, has been established by using the sub-supersolutions method in [26] or by other methods in [10]. These results have been extended to more general nonlinear terms, respectively, in [7, 18, 27], and [20].

In [20], the authors proved the existence and uniqueness of a positive continuous solution to the nonlinear elliptic problem

$$
\left\{\begin{array}{l}
\Delta u(x)+\varphi(\cdot, u)=0, \quad x \in \Omega(\text { in the distributional sense }), \\
u>0, \quad \text { in } \Omega, \\
u_{\mid \partial \Omega}=0 \\
\lim _{|x| \rightarrow \infty} u(x)=0,
\end{array}\right.
$$

where $\Omega$ is an unbounded domain in $\mathbb{R}^{n}(n \geq 3)$, with smooth boundary $\partial \Omega$ and $\varphi: \Omega \times$ $(0, \infty) \rightarrow(0, \infty)$ is continuous and nonincreasing with respect to the second variable, such that for all $c>0, V(\varphi(\cdot, c))>0$ and $\varphi(\cdot, c)$ belongs to $K_{n}^{\infty}(\Omega)$, where $V=(-\Delta)^{-1}$ and $K_{n}^{\infty}(\Omega)$ is the Kato class (see Definition 2.1).

In [4], the authors studied equation (1.1) on the whole space in the sublinear case. More precisely, they have proved the existence and uniqueness of the problem

$$
\left\{\begin{array}{l}
\Delta u(x)+p(x) u^{\gamma}=0, \quad x \in \mathbb{R}^{n}, n \geq 3 \\
u>0 \\
\liminf _{|x| \rightarrow \infty} u(x)=0,
\end{array}\right.
$$

where $0<\gamma<1$ and $p$ is a nonnegative measurable function such that the function $x \rightarrow$ $\int_{\mathbb{R}^{n}} \frac{p(y)}{|x-y|^{n-2}} d y$ belongs to $L^{\infty}\left(\mathbb{R}^{n}\right)$.

In [5], by using Karamata regular variation theory and the sub-supersolutions method, the authors studied the asymptotic behavior as $|x| \rightarrow \infty$ of the unique classical positive solution of problem (1.3) with $\gamma<1$ and $p(x)$ is a nonnegative function in $C_{\text {loc }}^{\alpha}\left(\mathbb{R}^{n}\right), 0<\alpha<$ 1 , such that there exists $c>0$ satisfying

$$
\frac{1}{c} \frac{L(1+|x|)}{(1+|x|)^{\lambda}} \leq p(x) \leq c \frac{L(1+|x|)}{(1+|x|)^{\lambda}}
$$

where $\lambda \geq 2$ and $L$ belongs to the class of slowly varying functions at infinity (see Definition 1.1).

In [1], the authors considered equation (1.1) in a punctured bounded domain. Under some sufficient conditions on the function $p(x)$, the existence of a positive continuous solution with a global behavior is obtained. Their approach is based on the Karamata regular variation theory and the Schauder fixed-point theorem.

The initial Karamata regular variation theory was developed by Karamata in [14]. In [8], the authors have shown that the class of Karamata regular variation functions is a well-suited framework for asymptotic analysis near the boundary for semilinear elliptic problems. For more works related to the Karamata regular variation theory, we refer the reader to $[15-17,19,22,24]$ and the reference therein. 
Motivated by the approach used in [1] and [5], in this paper, we consider the existence and global asymptotic behavior of a positive continuous solution to the following problem

$$
\left\{\begin{array}{l}
\Delta u(x)+p(x) u^{\gamma}=0, \quad x \in D \text { (in the distributional sense) } \\
u>0, \quad \text { in } D \\
\lim _{|x| \rightarrow 0}|x|^{n-2} u(x)=0, \\
\lim _{|x| \rightarrow \infty} u(x)=0,
\end{array}\right.
$$

where $\gamma<1, D=\mathbb{R}^{n} \backslash\{0\}(n \geq 3)$ and the potential function $p(x)$ is required to satisfy some convenient comparable asymptotic rate related to the class of slowly varying functions defined as follows; see for example [2, 14, 21, 25]:

Definition 1.1 A positive continuously differentiable function $L$ defined on $[A, \infty)$, for some $A>0$ is said to be normalized slowly varying (at infinity) if,

$$
\lim _{t \rightarrow \infty} t \frac{L^{\prime}(t)}{L(t)}=0
$$

we write $L \in \mathcal{N S} \mathcal{V}_{\infty}$.

As examples, we quote:

- $L(t)=\prod_{k=1}^{m}\left(\ln _{k} t\right)^{\xi_{k}}$, where $\ln _{k} t=\ln _{\ln } \ln _{k-1} t$ and $\xi_{k} \in \mathbb{R}$.

- $L(t)=\exp \left(\prod_{k=1}^{m}\left(\ln _{k} t\right)^{v_{k}}\right)$, where $0<v_{k}<1$.

- $L(t)=\exp \left\{(\ln t)^{\frac{1}{3}} \cos (\ln t)^{\frac{1}{3}}\right\}$.

The last example shows that the behavior at infinity for a slowly varying function cannot be predicted. Indeed, it exhibits "infinite oscillation" in the sense that

$$
\liminf _{t \rightarrow \infty} L(t)=0 \quad \text { and } \quad \limsup _{t \rightarrow \infty} L(t)=\infty
$$

On the other hand, the growth or decay of a slowly varying function as $t \rightarrow \infty$ is limited in the sense that it satisfies for any $\varepsilon>0$

$$
\lim _{t \rightarrow \infty} t^{\varepsilon} L(t)=\infty, \quad \lim _{t \rightarrow \infty} t^{-\varepsilon} L(t)=0 .
$$

Similarly, a class of normalized slowly varying (at zero) function is defined as follows:

Definition 1.2 A positive continuously differentiable function $L$ defined on $(0, a]$, for some $a>0$, is said to be normalized slowly varying (at zero) if,

$$
\lim _{t \rightarrow 0^{+}} t \frac{L^{\prime}(t)}{L(t)}=0
$$

we write $L \in \mathcal{N S} \mathcal{V}_{0}$.

Remark 1.3 Note that $L$ belongs to $\mathcal{N S} \mathcal{V}_{0}$ if and only if $t \rightarrow L(1 / t)$ belongs to $\mathcal{N S} \mathcal{V}_{\infty}$.

Throughout this paper, we make the following assumption: 
(H) $p$ is a positive continuous function in $D$ such that there exists $c>0$ satisfying

$$
{ }_{c}^{1} \mathcal{P}(x) \leq p(x) \leq c \mathcal{P}(x), \quad \text { for } x \in D,
$$

where $\mathcal{P}(x):=|x|^{-\mu} \mathcal{L}_{0}(\min (|x|, 1))(|x|+1)^{\mu-\lambda} \mathcal{L}_{\infty}(\max (|x|, 1))$, with $\gamma<1, \mu \leq n+(2-n) \gamma$ and $\lambda \geq 2$.

Here, $\mathcal{L}_{0} \in \mathcal{N} \mathcal{S} \mathcal{V}_{0}$, defined on $(0, a]$, for some $a>1$ and $\mathcal{L}_{\infty} \in \mathcal{N} \mathcal{S} \mathcal{V}_{\infty}$, defined on $[1, \infty)$ such that

$$
\int_{0}^{a} s^{n+(2-n) \gamma-\mu-1} \mathcal{L}_{0}(s) d s<\infty \text { and } \int_{1}^{\infty} s^{1-\lambda} \mathcal{L}_{\infty}(s) d s<\infty .
$$

Note that the comparable asymptotic rate of $p(x)$ in (1.7) determines the asymptotic behavior of the solution.

Our main result is summarized in the following theorem.

Theorem 1.4 Under assumption $(\mathrm{H})$, problem (1.5) has at least one positive continuous solution $u$ on $D$ such that

$$
\frac{1}{c} \theta(x) \leq u(x) \leq c \theta(x)
$$

where $c$ is a positive constant and for $x \in D$,

$$
\theta(x):=|x|^{\xi}\left(\widetilde{\mathcal{L}}_{0}(\min (|x|, 1))\right)^{\frac{1}{1-\gamma}}(|x|+1)^{\zeta-\xi}\left(\widetilde{\mathcal{L}}_{\infty}(\max (|x|, 1))\right)^{\frac{1}{1-\gamma}}
$$

where $\xi=\min \left(0, \frac{2-\mu}{1-\gamma}\right), \zeta=\max \left(2-n, \frac{2-\lambda}{1-\gamma}\right)$ and $\widetilde{\mathcal{L}}_{0} \in \mathcal{N} \mathcal{S} \mathcal{V}_{0}\left(\right.$ resp., $\left.\widetilde{\mathcal{L}}_{\infty} \in \mathcal{N} \mathcal{S} \mathcal{V}_{\infty}\right)$ is defined on $(0, a)($ resp., on $[1, \infty))$ by

$$
\widetilde{\mathcal{L}}_{0}(t):= \begin{cases}1, & \text { if } \mu<2, \\ \int_{t}^{a} \frac{\mathcal{L}_{0}(s)}{s} d s, & \text { if } \mu=2, \\ \mathcal{L}_{0}(t), & \text { if } 2<\mu<n+(2-n) \gamma \\ \int_{0}^{t} \frac{\mathcal{L}_{0}(s)}{s} d s, & \text { if } \mu=n+(2-n) \gamma\end{cases}
$$

and

$$
\tilde{\mathcal{L}}_{\infty}(t):= \begin{cases}\int_{t+1}^{\infty} \frac{\mathcal{L}_{\infty}(s)}{s} d s, & \text { if } \lambda=2, \\ \mathcal{L}_{\infty}(t+1), & \text { if } 2<\lambda<n+(2-n) \gamma, \\ \int_{1}^{t+1} \frac{\mathcal{L}_{\infty}(s)}{s} d s, & \text { if } \lambda=n+(2-n) \gamma, \\ 1, & \text { if } \lambda>n+(2-n) \gamma .\end{cases}
$$

Remark 1.5 From (1.9) and (1.6), we obtain

$$
\lim _{|x| \rightarrow 0} u(x)=\infty, \quad \text { for } \mu>2 .
$$

That is, the solution blows-up at the origin. 
The outline of this article is as follows. In Sect. 2, we prove some pertinent properties related to the Kato class and also to the Karamata regular variation theory. In Sect. 3, we show the existence of a solution to problem (1.5) with the required asymptotic behavior (1.9).

In this paper, we use the following notations:

(i) $D=\mathbb{R}^{n} \backslash\{0\}(n \geq 3)$.

(ii) $\mathcal{B}(D)$ denotes the set of all Borel measurable functions in $D$ and $\mathcal{B}^{+}(D)$ denotes the set of nonnegative ones.

(iii) $C(D)$ refers to all continuous functions in $D$.

(iv) $s \wedge t=\min (s, t)$ and $s \vee t=\max (s, t)$, for all $s, t \in \mathbb{R}$.

(v) For $f, g \in \mathcal{B}^{+}(D), f \approx g$ in $D$, means that there exists $c>0$ such that $\frac{1}{c} f(x) \leq g(x) \leq c f(x)$, for all $x \in D$.

(vi) $\mathcal{S}^{+}(\Omega)$ denotes the set of all nonnegative superharmonic functions on an open set $\Omega$ of $\mathbb{R}^{n}$.

(vii) For $x \in D$,

$$
\varrho_{0}(x):=\frac{1+|x|^{n-2}}{|x|^{n-2}} .
$$

Note that $\varrho_{0} \in \mathcal{S}^{+}\left(\mathbb{R}^{n}\right)$ and harmonic on $D$, see, for example, [3].

(viii) For $x, y \in \mathbb{R}^{n}$, we denote the normalized fundamental solution of Laplace's equation by:

$$
\Gamma(x, y)=\frac{c_{n}}{|x-y|^{n-2}}, \quad \text { with } c_{n}=\frac{\Gamma\left(\frac{n}{2}-1\right)}{4 \pi^{\frac{n}{2}}} .
$$

(ix) The Newtonian potential $\mathcal{N}$ is defined on $\mathcal{B}^{+}(D)$ by

$$
\mathcal{N} f(x)=\int_{D} \Gamma(x, y) f(y) d y
$$

From [6, Proposition 2.10], we learned that if $f \in \mathcal{B}^{+}(D)$ such that $f \in L_{\text {loc }}^{1}(D)$ and $\mathcal{N} f \in$ $L_{\text {loc }}^{1}(D)$, then

$-\Delta(\mathcal{N} f)=f, \quad$ in $D$ (in the distributional sense).

Throughout this paper, the letter $c$ will denote a generic positive constant that may vary from line to line.

\section{Preliminaries}

2.1 Kato class $K_{n}^{\infty}(D)$

Definition 2.1 (See [28]) A function $\psi$ in $\mathcal{B}(D)$ is said to be in the Kato class $K_{n}^{\infty}(D)$ if

$$
\lim _{r \rightarrow 0}\left(\sup _{x \in D} \int_{D \cap B(x, r)} \Gamma(x, y)|\psi(y)| d y\right)=0,
$$

and

$$
\lim _{M \rightarrow \infty}\left(\sup _{x \in D} \int_{D \cap(|y| \geq M)} \Gamma(x, y)|\psi(y)| d y\right)=0,
$$

where $\Gamma(x, y)$ is given by $(1.13)$. 
Example 2.2 Let $p>\frac{n}{2}$. Then, we have

$$
L^{p}(D) \cap L^{1}(D) \subset K_{n}^{\infty}(D)
$$

Indeed, for $\psi \in L^{p}(D)$, by using the Hölder inequality, it is clear that (2.1) holds. Now, assume further that $\psi \in L^{1}(D)$, then

$$
\begin{aligned}
& \int_{D \cap(|y| \geq M)} \Gamma(x, y)|\psi(y)| d y \\
& \quad \leq \int_{D \cap B(x, r)} \Gamma(x, y)|\psi(y)| d y+c_{n} r^{2-n} \int_{D \cap(|y| \geq M) \cap(|x-y| \geq r)}|\psi(y)| d y .
\end{aligned}
$$

Hence, $\psi$ satisfies (2.2).

The next Lemma is due to Mâagli and Zribi, see [20, Remark 2 and Proposition 1].

\section{Lemma 2.3}

(i) Let $\psi$ be a radial function in $D$, then

$$
\psi \in K_{n}^{\infty}(D) \quad \text { if and only if } \quad \int_{0}^{\infty} r|\psi(r)| d r<\infty .
$$

(ii) Let $\psi \in \mathcal{B}(D)$ satisfying (2.1). Then, for each $M>0$, we have

$$
\int_{D \cap(|y| \leq M)}|\psi(y)| d y<\infty
$$

Remark 2.4 For all $x, y, z \in \mathbb{R}^{n}$, we have

$$
\frac{\Gamma(x, y) \Gamma(y, z)}{\Gamma(x, z)} \leq 2^{n-3} c_{n}(\Gamma(x, y)+\Gamma(y, z)),
$$

where $c_{n}=\frac{\Gamma\left(\frac{n}{2}-1\right)}{4 \pi^{\frac{n}{2}}}$.

Proposition 2.5 Let $\psi \in K_{n}^{\infty}(D), x_{0} \in \mathbb{R}^{n}$ and $h \in \mathcal{S}^{+}(D)$. Then, we have

$$
\lim _{r \rightarrow 0}\left(\sup _{x \in D} \frac{1}{h(x)} \int_{D \cap B\left(x_{0}, r\right)} \Gamma(x, y) h(y)|\psi(y)| d y\right)=0,
$$

and

$$
\lim _{M \rightarrow \infty}\left(\sup _{x \in D} \frac{1}{h(x)} \int_{D \cap(|y| \geq M)} \Gamma(x, y) h(y)|\psi(y)| d y\right)=0 .
$$

Proof Since $h \in \mathcal{S}^{+}(D)$, then by [23, Theorem 2.1, p. 164], there exists a sequence $\left(h_{k}\right)_{k} \subset$ $\mathcal{B}^{+}(D)$ such that

$$
h(y)=\sup _{k} \int_{D} \Gamma(y, z) h_{k}(z) d y, \quad \text { for } y \in D .
$$

Therefore, we need to prove (2.4) and (2.5) only for $h(y)=\Gamma(y, z)$ uniformly in $z \in D$. 
Let $r>0$. By using Remark 2.4, there exists a constant $c>0$, such that for all $x, y, z \in D$,

$$
\frac{1}{h(x)} \int_{D \cap B\left(x_{0}, r\right)} \Gamma(x, y) h(y)|\psi(y)| d y \leq 2 c \sup _{\xi \in D} \int_{D \cap B\left(x_{0}, r\right)} \Gamma(\xi, y)|\psi(y)| d y .
$$

For $\varepsilon>0$, by Definition 2.1, there exists $s>0$ and $M>0$ such that

$$
\int_{D \cap B\left(x_{0}, r\right)} \Gamma(\xi, y)|\psi(y)| d y \leq \varepsilon+\frac{c_{n}}{s^{n-2}} \int_{D \cap B\left(x_{0}, r\right) \cap(|y| \leq M)}|\psi(y)| d y .
$$

Using this fact, (2.6) and Lemma 2.3(ii), we obtain (2.4) by letting $r \rightarrow 0$.

Finally, note that assertion (2.5) follows by using similar arguments as above.

Proposition 2.6 Let $\psi \in K_{n}^{\infty}(D)$ and $\varrho_{0}(x):=\frac{1+|x|^{n-2}}{|x|^{n-2}}$. Then, the function

$$
v(x):=\frac{1}{\varrho_{0}(x)} \int_{D} \Gamma(x, y) \varrho_{0}(y) \psi(y) d y
$$

is continuous on $\mathbb{R}^{n}$ with $\lim _{|x| \rightarrow \infty} v(x)=0$. That is, $v(x) \in C_{0}\left(\mathbb{R}^{n}\right)$.

Proof Let $\psi \in K_{n}^{\infty}(D)$ and $x_{0} \in \mathbb{R}^{n}$. Since $\varrho_{0} \in \mathcal{S}^{+}(D)$, then for $\varepsilon>0$, by Proposition 2.5, there exists $M>r>0$, such that the following holds:

(i) If $x_{0} \neq 0$, then for $x \in B\left(x_{0}, \frac{r}{2}\right) \cap D$, we have

$$
\left|v(x)-v\left(x_{0}\right)\right| \leq \frac{\varepsilon}{2}+\int_{D_{0} \cap(|y| \leq M)}\left|\frac{1}{\varrho_{0}(x)} \Gamma(x, y)-\frac{1}{\varrho_{0}\left(x_{0}\right)} \Gamma\left(x_{0}, y\right)\right| \varrho_{0}(y)|\psi(y)| d y
$$

where $D_{0}=D \cap B^{c}(0, r) \cap B^{c}\left(x_{0}, r\right)$.

Since $(x, y) \mapsto \frac{1}{\varrho_{0}(x)} \Gamma(x, y)$ is continuous on $\left(B\left(x_{0}, \frac{r}{2}\right) \cap D\right) \times\left(D_{0} \cap(|y| \leq M)\right)$, we obtain by Lemma 2.3 (ii) and Lebesgue's dominated convergence theorem,

$$
\int_{D_{0} \cap(|y| \leq M)}\left|\frac{1}{\varrho_{0}(x)} \Gamma(x, y)-\frac{1}{\varrho_{0}\left(x_{0}\right)} \Gamma\left(x_{0}, y\right)\right| \varrho_{0}(y)|\psi(y)| d y \rightarrow 0 \quad \text { as } x \rightarrow x_{0} .
$$

Hence, there exists $\delta>0$ with $\delta<\frac{r}{2}$ such that if $x \in B\left(x_{0}, \delta\right) \cap D$,

$$
\int_{D_{0} \cap(|y| \leq M)}\left|\frac{1}{\varrho_{0}(x)} \Gamma(x, y)-\frac{1}{\varrho_{0}\left(x_{0}\right)} \Gamma\left(x_{0}, y\right)\right| \varrho_{0}(y)|\psi(y)| d y \leq \frac{\varepsilon}{2} .
$$

Hence, for $x \in B\left(x_{0}, \delta\right) \cap D$, we have

$$
\left|v(x)-v\left(x_{0}\right)\right| \leq \varepsilon
$$

That is,

$$
\lim _{x \rightarrow x_{0}} v(x)=v\left(x_{0}\right)
$$

(ii) If $x_{0}=0$ and $x \in B\left(0, \frac{r}{2}\right) \cap D$, then we have

$$
|v(x)| \leq \frac{\varepsilon}{2}+\int_{D \cap B^{c}(0, r) \cap(|y| \leq M)} \frac{1}{\varrho_{0}(x)} \Gamma(x, y) \varrho_{0}(y)|\psi(y)| d y .
$$


Now, since $\lim _{|x| \rightarrow 0} \frac{1}{\varrho_{0}(x)} \Gamma(x, y) \varrho_{0}(y)=0$, for all $y \in D \cap B^{c}(0, r) \cap(|y| \leq M)$, we deduce by similar arguments as above that

$$
\lim _{|x| \rightarrow 0} v(x)=0=v\left(x_{0}\right)
$$

(iii) It remains to prove that $\lim _{|x| \rightarrow \infty} v(x)=0$.

To this end, let $x \in D$ such that $|x| \geq M+1$. Using Proposition 2.5 and Lemma 2.3 (ii), we deduce that

$$
\begin{aligned}
|v(x)| & \leq \frac{\varepsilon}{2}+\frac{1+M^{n-2}}{r^{n-2}} \int_{D \cap B^{c}(0, r) \cap(|y| \leq M)} \Gamma(x, y)|\psi(y)| d y \\
& \leq \frac{\varepsilon}{2}+\frac{c}{(|x|-M)^{n-2}}
\end{aligned}
$$

where $c$ is some positive constant.

This implies that $\lim _{|x| \rightarrow \infty} v(x)=0$.

\subsection{Karamata regular variation theory}

Let us recall some basic properties of Karamata regular variation theory (see [2, 14, 21, 24, 25]).

The following result concerns operations that preserve slow variation.

Proposition 2.7 If $L_{1}(t), L_{2}(t)$ are slowly varying at infinity (resp., at zero), then the same holds for $L_{1}(t)+L_{2}(t), L_{1}(t) L_{2}(t)$, and $\left(L_{1}(t)\right)^{v}$ for any $v \in \mathbb{R}$.

\section{Proposition 2.8}

(i) If $L(t) \in \mathcal{N S} \mathcal{V}_{\infty}$, then for any $\varepsilon>0$

$$
\lim _{t \rightarrow \infty} t^{\varepsilon} L(t)=\infty, \quad \lim _{t \rightarrow \infty} t^{-\varepsilon} L(t)=0
$$

(ii) If $L(t) \in \mathcal{N S} \mathcal{V}_{0}$, then for any $\varepsilon>0$

$$
\lim _{t \rightarrow 0^{+}} t^{\varepsilon} L(t)=0 \quad \text { and } \quad \lim _{t \rightarrow 0^{+}} t^{-\varepsilon} L(t)=\infty .
$$

The following result, termed Karamata's integration theorem, will be used later.

Proposition 2.9 Let $L(t) \in \mathcal{N S} \mathcal{V}_{\infty}$. Then,

(i) if $v>-1$,

$$
\int_{A}^{t} s^{v} L(s) d s \sim \frac{1}{v+1} t^{v+1} L(t), \quad t \rightarrow \infty
$$

(ii) if $v<-1$,

$$
\int_{t}^{\infty} s^{v} L(s) d s \sim-\frac{1}{v+1} t^{v+1} L(t), \quad t \rightarrow \infty
$$


(iii) if $v=-1$,

$$
l(t)=\int_{A}^{t} s^{-1} L(s) d s \in \mathcal{N} \mathcal{S} \mathcal{V}_{\infty} \quad \text { and } \quad \lim _{t \rightarrow \infty} \frac{L(t)}{l(t)}=0
$$

and if $\int_{A}^{\infty} s^{-1} L(s) d s<\infty$,

$$
m(t)=\int_{t}^{\infty} s^{-1} L(s) d s \in \mathcal{N} \mathcal{S} \mathcal{V}_{\infty} \quad \text { and } \quad \lim _{t \rightarrow \infty} \frac{L(t)}{m(t)}=0
$$

The following is an analog of Proposition 2.9 for $L$ defined at zero instead of $\infty$.

Proposition 2.10 Let $L(t) \in \mathcal{N} \mathcal{S} \mathcal{V}_{0}$. Then,

(i) if $v>-1$,

$$
\int_{0}^{t} s^{v} L(s) d s \sim \frac{1}{v+1} t^{v+1} L(t), \quad t \rightarrow 0^{+}
$$

(ii) if $v<-1$,

$$
\int_{t}^{a} s^{v} L(s) d s \sim-\frac{1}{v+1} t^{v+1} L(t), \quad t \rightarrow 0^{+}
$$

(iii) if $v=-1$,

$$
l_{0}(t)=\int_{t}^{a} s^{-1} L(s) d s \in \mathcal{N} \mathcal{S} \mathcal{V}_{0} \quad \text { and } \quad \lim _{t \rightarrow 0^{+}} \frac{L(t)}{l_{0}(t)}=0
$$

and if $\int_{0}^{a} s^{-1} L(s) d s<\infty$,

$$
m_{0}(t)=\int_{0}^{t} s^{-1} L(s) d s \in \mathcal{N} \mathcal{S} \mathcal{V}_{0} \quad \text { and } \quad \lim _{t \rightarrow 0^{+}} \frac{L(t)}{m_{0}(t)}=0
$$

The following result, will play a central role in establishing our main result in Sect. 3.

Proposition 2.11 For $\alpha \leq n$ and $\beta \geq 2$, set

$$
b(x)=|x|^{-\alpha} L_{0}(|x| \wedge 1)(|x|+1)^{\alpha-\beta} L_{\infty}(|x| \vee 1), \quad x \in D,
$$

where $L_{0} \in \mathcal{N S} \mathcal{V}_{0}$ defined on $(0, a]$, for some $a>1$ and $L_{\infty} \in \mathcal{N} \mathcal{S} \mathcal{V}_{\infty}$, defined on $[1, \infty)$ such that

$$
\int_{0}^{a} s^{n-\alpha-1} L_{0}(s) d s<\infty \quad \text { and } \quad \int_{1}^{\infty} s^{1-\beta} L_{\infty}(s) d s<\infty
$$

Then,

$$
\mathcal{N} b(x) \approx|x|^{\min (0,2-\alpha)} \widetilde{L}_{0}(|x| \wedge 1)(|x|+1)^{\max (2-n, 2-\beta)-\min (0,2-\alpha)} \widetilde{L}_{\infty}(|x| \vee 1), \quad \text { on } D
$$


where for $t \in(0, a)$,

$$
\widetilde{L}_{0}(t):= \begin{cases}1, & \text { if } \alpha<2, \\ \int_{t}^{a} \frac{L_{0}(s)}{s} d s, & \text { if } \alpha=2, \\ L_{0}(t), & \text { if } 2<\alpha<n, \\ \int_{0}^{t} \frac{L_{0}(s)}{s} d s, & \text { if } \alpha=n,\end{cases}
$$

and for $t \geq 1$,

$$
\widetilde{L}_{\infty}(t):= \begin{cases}1, & \text { if } \beta>n, \\ \int_{1}^{t+1} \frac{L_{\infty}(s)}{s} d s, & \text { if } \beta=n, \\ L_{\infty}(t+1), & \text { if } 2<\beta<n, \\ \int_{t+1}^{\infty} \frac{L_{\infty}(s)}{s} d s, & \text { if } \beta=2 .\end{cases}
$$

Proof Since $b$ is a nonnegative radial measurable function on $D$, it follows from [23, Proposition 1.7], that

$$
\mathcal{N} b(x):=\int_{D} \Gamma(x, y) b(y) d y=c \int_{0}^{\infty} \frac{r^{n-1}}{(|x| \vee r)^{n-2}} b(r) d r=: c J(|x|),
$$

where the function $J$ is defined on $[0, \infty)$ by

$$
J(t)=\int_{0}^{\infty} \frac{r^{n-\alpha-1}}{(t \vee r)^{n-2}}(r+1)^{\alpha-\beta} L_{0}(r \wedge 1) L_{\infty}(r \vee 1) d r .
$$

We need to estimate $J(t)$. Note that, under condition $(2.7), J(t)<\infty$.

Let $a>1$, then we have

$$
\begin{aligned}
J(t) & \approx \int_{0}^{a} \frac{r^{n-\alpha-1}}{(t \vee r)^{n-2}} L_{0}(r) d r+\int_{a}^{\infty} \frac{r^{n-\beta-1}}{(t \vee r)^{n-2}} L_{\infty}(r) d r \\
& :=J_{1}(t)+J_{2}(t) .
\end{aligned}
$$

We discuss the following cases:

Case $1.0<t \leq 1$. Clearly from (2.7), we have

$$
J_{2}(t)=\int_{a}^{\infty} r^{1-\beta} L_{\infty}(r) d r \approx 1
$$

On the other hand, by writing

$$
J_{1}(t)=t^{2-n} \int_{0}^{t} r^{n-\alpha-1} L_{0}(r) d r+\int_{t}^{a} r^{1-\alpha} L_{0}(r) d r,
$$

we deduce that

$$
J(t) \approx t^{2-n} \int_{0}^{t} r^{n-\alpha-1} L_{0}(r) d r+\left(1+\int_{t}^{a} r^{1-\alpha} L_{0}(r) d r\right) .
$$


Therefore, by (2.7) and Propositions 2.8 and 2.10, we obtain

$$
J(t) \approx \phi_{0}(t):= \begin{cases}1, & \text { if } \alpha<2, \\ \int_{t}^{a} \frac{L_{0}(r)}{r} d r, & \text { if } \alpha=2, \\ t^{2-\alpha} L_{0}(t), & \text { if } 2<\alpha<n \\ t^{2-\alpha} \int_{0}^{t} \frac{L_{0}(r)}{r} d r, & \text { if } \alpha=n\end{cases}
$$

That is,

$$
J(t) \approx t^{\min (0,2-\alpha)} \widetilde{L}_{0}(t), \quad \text { for } 0<t \leq 1
$$

Case 2. $t \geq a+1$. From (2.7), we derive that

$$
J_{1}(t) \approx t^{2-n} \int_{0}^{a} r^{n-\alpha-1} L_{0}(r) d r \approx t^{2-n}
$$

On the other hand, since

$$
J_{2}(t)=t^{2-n} \int_{a}^{t} r^{n-\beta-1} L_{\infty}(r) d r+\int_{t}^{\infty} r^{1-\beta} L_{\infty}(r) d r
$$

we deduce that

$$
J(t) \approx t^{2-n}\left(1+\int_{a}^{t} r^{n-\beta-1} L_{\infty}(r) d r\right)+\int_{t}^{\infty} r^{1-\beta} L_{\infty}(r) d r .
$$

Hence, by (2.7) and Propositions 2.8 and 2.9, we obtain

$$
J(t) \approx \begin{cases}t^{2-n}, & \text { if } \beta>n \\ t^{2-n} \int_{a}^{t} \frac{L_{\infty}(s)}{s} d s, & \text { if } \beta=n \\ t^{2-\beta} L_{\infty}(t), & \text { if } 2<\beta<n \\ \int_{t}^{\infty} \frac{L_{\infty}(s)}{s} d s, & \text { if } \beta=2 .\end{cases}
$$

Therefore, by using Proposition 2.9 and [5, Lemma 2.3], we conclude that

$$
J(t) \approx \phi_{\infty}(t):= \begin{cases}(t+1)^{2-n}, & \text { if } \beta>n, \\ (t+1)^{2-n} \int_{1}^{t+1} \frac{L_{\infty}(s)}{s} d s, & \text { if } \beta=n, \\ (t+1)^{2-\beta} L_{\infty}(t+1), & \text { if } 2<\beta<n, \\ \int_{t+1}^{\infty} \frac{L_{\infty}(s)}{s} d s, & \text { if } \beta=2 .\end{cases}
$$

Hence,

$$
J(t) \approx(t+1)^{\max (2-n, 2-\beta)} \widetilde{L}_{\infty}(t), \quad \text { for } t \geq a+1
$$

Finally, since $J(t), \phi_{0}(t)$ and $\phi_{\infty}(t)$ are positive continuous functions on $[1, a+1]$, we deduce that

$$
J(t) \approx \phi_{0}(t) \phi_{\infty}(t), \quad \text { on }[1, a+1]
$$


Hence, by combining (2.8), (2.9) and (2.10), we obtain

$$
J(t) \approx t^{\min (0,2-\alpha)} \widetilde{L}_{0}(t \wedge 1)(t+1)^{\max (2-n, 2-\beta)-\min (0,2-\alpha)} \widetilde{L}_{\infty}(t \vee 1), \quad \text { on }[0, \infty)
$$

This completes the proof.

Proposition 2.12 Assume that p satisfies hypothesis $(\mathrm{H})$, then

$$
\mathcal{N}\left(p \theta^{\gamma}\right)(x) \approx \theta(x), \quad \text { on } D
$$

where $\gamma<1$ and $\theta$ is given in (1.10).

Proof Using (1.7) and (1.10), we obtain

$$
\begin{aligned}
& p(x) \theta^{\gamma}(x) \\
& \quad \approx|x|^{-\alpha} \mathcal{L}_{0}(|x| \wedge 1)\left(\widetilde{\mathcal{L}}_{0}(\min (|x|, 1))\right)^{\frac{\gamma}{1-\gamma}}(|x|+1)^{\alpha-\beta} \mathcal{L}_{\infty}(|x| \vee 1)\left(\widetilde{\mathcal{L}}_{\infty}(|x| \vee 1)\right)^{\frac{\gamma}{1-\gamma}}
\end{aligned}
$$

where $\alpha:=\mu-\gamma \min \left(0, \frac{2-\mu}{1-\gamma}\right)$ and $\beta:=\lambda-\gamma \max \left(2-n, \frac{2-\lambda}{1-\gamma}\right)$.

From the fact that $\mu \leq n+(2-n) \gamma$ and $\lambda \geq 2$, we derive that $\alpha \leq n$ and $\beta \geq 2$.

By using the basic properties of Karamata regular variation theory and Proposition 2.11 with $L_{0}=\mathcal{L}_{0}(|x| \wedge 1)\left(\widetilde{\mathcal{L}}_{0}(\min (|x|, 1))\right)^{\frac{\gamma}{1-\gamma}} \in \mathcal{N} \mathcal{S} \mathcal{V}_{0}$ and $L_{\infty}=\mathcal{L}_{\infty}(|x| \vee 1)\left(\widetilde{\mathcal{L}}_{\infty}(|x| \vee 1)\right)^{\frac{\gamma}{1-\gamma}} \in$ $\mathcal{N S} \mathcal{V}_{\infty}$, we deduce that

$$
\mathcal{N}\left(p \theta^{\gamma}\right)(x) \approx|x|^{\min (0,2-\alpha)} \widetilde{L}_{0}(|x| \wedge 1)(|x|+1)^{\max (2-n, 2-\beta)-\min (0,2-\alpha)} \widetilde{L}_{\infty}(|x| \vee 1)
$$

Since $\min (0,2-\alpha)=\min \left(0, \frac{2-\mu}{1-\gamma}\right):=\xi$ and $\max (2-n, 2-\beta)=\max \left(2-n, \frac{2-\lambda}{1-\gamma}\right):=\zeta$, we deduce that

$$
\mathcal{N}\left(p \theta^{\gamma}\right)(x) \approx|x|^{\xi} \widetilde{L}_{0}(|x| \wedge 1)(|x|+1)^{\zeta-\xi} \widetilde{L}_{\infty}(|x| \vee 1) \approx \theta(x)
$$

This completes the proof.

\section{Proof of Theorem 1.4}

In order to prove Theorem 1.4, we need to establish some preliminary results. Our approach is inspired from methods developed in [20] with necessary modifications.

For $v>0$, we denote by $\left(P_{v}\right)$ the following problem

$$
\left(P_{\nu}\right)\left\{\begin{array}{l}
\Delta u(x)+p(x) u^{\gamma}(x)=0, \quad x \in D \text { (in the distributional sense), } \\
u>0 \text { in } D \\
\lim _{|x| \rightarrow 0}|x|^{n-2} u(x)=v \\
\lim _{|x| \rightarrow \infty} u(x)=v .
\end{array}\right.
$$

We recall that for $x \in D, \varrho_{0}(x)=\frac{1+|x|^{n-2}}{|x|^{n-2}}$. 
Note that $v \varrho_{0}(x)$ is a solution of the following homogeneous problem

$$
\left(H_{v}\right)\left\{\begin{array}{l}
\Delta u(x)=0, \quad x \in D, \\
u>0 \quad \text { in } D, \\
\lim _{|x| \rightarrow 0}|x|^{n-2} u(x)=v, \\
\lim _{|x| \rightarrow \infty} u(x)=v .
\end{array}\right.
$$

Problem $\left(P_{v}\right)$ can be seen as a perturbation of problem $\left(H_{v}\right)$.

Proposition 3.1 Let $\gamma<0$ and assume that hypothesis $(\mathrm{H})$ is satisfied. Then, for each $v>0$, problem $\left(P_{v}\right)$ has at least one positive solution $u_{v} \in C(D \cup\{\infty\})$ satisfying for $x \in D$

$$
u_{v}(x)=v \varrho_{0}(x)+\int_{D} \Gamma(x, y) p(y) u_{v}^{\gamma}(y) d y
$$

In particular,

$$
u_{v}(x) \approx \varrho_{0}(x), \quad \text { on } D \text {. }
$$

Proof Let $\gamma<0$ and $v>0$. Due to Lemma 2.3 (i) and hypothesis $(H)$, the function $\psi(y):=$ $\left(\varrho_{0}(y)\right)^{(\gamma-1)} p(y)$ becomes in $K_{n}^{\infty}(D)$.

Therefore, by Proposition 2.6, we have

$$
x \mapsto h(x):=\frac{1}{\varrho_{0}(x)} \int_{D} \Gamma(x, y)\left(\varrho_{0}(y)\right)^{\gamma} p(y) d y \in C_{0}\left(\mathbb{R}^{n}\right) .
$$

Let $\beta_{0}:=v+v^{\gamma}\|h\|_{\infty}$ and consider the convex set $\Lambda$ given by

$$
\Lambda=\left\{\vartheta \in C\left(\mathbb{R}^{n} \cup\{\infty\}\right): v \leq \vartheta \leq \beta_{0}\right\} .
$$

Define the operator $T$ on $\Lambda$ by

$$
T \vartheta(x)=v+\frac{1}{\varrho_{0}(x)} \int_{D} \Gamma(x, y) p(y)\left(\varrho_{0}(y)\right)^{\gamma} \vartheta^{\gamma}(y) d y .
$$

Since for all $\vartheta \in \Lambda, \vartheta^{\gamma} \leq v^{\gamma}$, then as in the proof of Proposition 2.6 we show that the family $T \Lambda$ is equicontinuous in $\mathbb{R}^{n} \cup\{\infty\}$. In particular, for all $\vartheta \in \Lambda, T \vartheta \in C\left(\mathbb{R}^{n} \cup\{\infty\}\right)$ and so $T \Lambda \subset \Lambda$.

Moreover, the family $\{T \vartheta(x), \vartheta \in \Lambda\}$ is uniformly bounded in $\mathbb{R}^{n} \cup\{\infty\}$, then by the Arzela-Ascoli theorem (see, for example [9, p. 62]) the set $T(\Lambda)$ becomes relatively compact in $C\left(\mathbb{R}^{n} \cup\{\infty\}\right)$.

To prove the continuity of $T$ in $\Lambda$, we consider a sequence $\left(\vartheta_{k}\right)_{k} \subset \Lambda$ and $\vartheta \in \Lambda$ such that $\left\|\vartheta_{k}-\vartheta\right\|_{\infty} \rightarrow 0$ as $k \rightarrow \infty$. Then, we have

$$
\left|T \vartheta_{k}(x)-T \vartheta(x)\right| \leq \frac{1}{\varrho_{0}(x)} \int_{D} \Gamma(x, y) p(y)\left(\varrho_{0}(y)\right)^{\gamma}\left|\vartheta_{k}^{\gamma}(y)-\vartheta^{\gamma}(y)\right| d y .
$$

Now, since

$$
\left|\vartheta_{k}^{\gamma}(y)-\vartheta^{\gamma}(y)\right| \leq 2 v^{\gamma},
$$


we deduce by the dominated convergence theorem and Proposition 2.6 that

$$
\forall x \in \mathbb{R}^{n} \cup\{\infty\}, \quad T \vartheta_{k}(x) \rightarrow T \vartheta(x) \quad \text { as } k \rightarrow \infty
$$

Since $T(\Lambda)$ is relatively compact in $C\left(\mathbb{R}^{n} \cup\{\infty\}\right)$, we obtain

$$
\left\|T \vartheta_{k}-T \vartheta\right\|_{\infty} \rightarrow 0 \quad \text { as } k \rightarrow \infty
$$

Hence, $T$ is a compact mapping of $\Lambda$ to itself and by the Schauder fixed-point theorem, there exists $\vartheta_{v} \in \Lambda$ such that for each $x \in \mathbb{R}^{n}$

$$
\vartheta_{v}(x)=v+\frac{1}{\varrho_{0}(x)} \int_{D} \Gamma(x, y) p(y)\left(\varrho_{0}(y)\right)^{\gamma} \vartheta_{v}^{\gamma}(y) d y .
$$

Since $\vartheta_{v}^{\gamma} \leq v^{\gamma}$, we deduce from (3.3) and (3.2) that

$$
\lim _{|x| \rightarrow \infty} \vartheta_{v}(x)=v \quad \text { and } \quad \lim _{|x| \rightarrow 0} \vartheta_{v}(x)=\vartheta_{v}(0)=v
$$

Put $u_{v}(x)=\varrho_{0}(x) \vartheta_{v}(x)$, for $x \in D$. Then, $u_{v} \in C(D \cup\{\infty\})$ and we have

$$
u_{v}(x)=v \varrho_{0}(x)+\int_{D} \Gamma(x, y) p(y) u_{v}^{\gamma}(y) d y
$$

and

$$
v \varrho_{0}(x) \leq u_{v}(x) \leq \beta \varrho_{0}(x)
$$

Now, since the function $y \mapsto p(y) u_{\nu}^{\gamma}(y) \in L_{\text {loc }}^{1}(D)$ and from (3.5) the function $x \mapsto$ $\mathcal{N}\left(p u_{\nu}^{\gamma}\right)(x) \in L_{\text {loc }}^{1}(D)$, we deduce by (1.15) that $u_{\nu}$ satisfies

$-\Delta u_{v}(x)=p(x) u_{v}^{\gamma}(x), \quad x \in D$, (in the distributional sense).

By (3.4), we have

$$
\lim _{|x| \rightarrow 0}|x|^{n-2} u_{v}(x)=\lim _{|x| \rightarrow \infty} u_{v}(x)=v .
$$

This completes the proof.

The next result is due to Mâagli and Zribi, see [20, Lemma 1].

Lemma 3.2 Let $g \in \mathcal{B}^{+}(D)$ and $v \in \mathcal{S}^{+}(D)$. Then, for any $w \in \mathcal{B}(D)$ such that $\mathcal{N}(g|w|)<\infty$ and $w+\mathcal{N}(g w)=v$, we have

$$
0 \leq w \leq v
$$

Corollary 3.3 Let $\gamma<0,0<v_{1} \leq v_{2}$ and $u_{v_{i}} \in C(D \cup\{\infty\})$ be the solution of problem $\left(P_{v_{i}}\right)$ given by (3.1). Then, we have

$$
0 \leq u_{v_{2}}(x)-u_{v_{1}}(x) \leq\left(v_{2}-v_{1}\right) \varrho_{0}(x), \quad \text { for } x \in D .
$$


Proof Let $g$ be the function defined on $D$ by

$$
g(x)= \begin{cases}p(x) \frac{u_{\nu_{2}}^{\gamma}(x)-u_{\nu_{1}}^{\gamma}(x)}{u_{v_{1}}(x)-u_{v_{2}}(x)}, & \text { if } u_{\nu_{1}}(x) \neq u_{\nu_{2}}(x), \\ 0, & \text { if } u_{\nu_{1}}(x)=u_{\nu_{2}}(x) .\end{cases}
$$

Since $\gamma<0$, then $g \in \mathcal{B}^{+}(D)$ and by (3.1) we have

$$
u_{v_{2}}-u_{v_{1}}+\mathcal{N}\left(g\left(u_{v_{2}}-u_{v_{1}}\right)\right)=\left(v_{2}-v_{1}\right) \varrho_{0}(x)
$$

Using (3.6) and (3.2), we obtain for $x \in D$,

$$
\begin{aligned}
\mathcal{N}\left(g\left|u_{\nu_{2}}-u_{\nu_{1}}\right|\right)(x) & =\int_{D} \Gamma(x, y) p(y)\left|u_{v_{2}}^{\gamma}(y)-u_{v_{1}}^{\gamma}(y)\right| d y \\
& \leq\left(v_{1}^{\gamma}+v_{2}^{\gamma}\right) \int_{D} \Gamma(x, y) p(y)\left(\varrho_{0}(y)\right)^{\gamma} d y \\
& =\left(v_{1}^{\gamma}+v_{2}^{\gamma}\right) \varrho_{0}(x) h(x)<\infty .
\end{aligned}
$$

Hence, by (3.8) and Lemma 3.2 with $w=u_{v_{2}}-u_{\nu_{1}}$, we obtain (3.7).

Proposition 3.4 Let $\gamma<0$. Under hypothesis $(\mathrm{H})$, problem (1.5) has at least one positive solution $u \in C(D)$ satisfying for $x \in D$

$$
u(x)=\int_{D} \Gamma(x, y) p(y) u^{\gamma}(y) d y
$$

Proof Let $\left(v_{k}\right)_{k}$ be a positive sequence decreasing to zero. Let $u_{k} \in C(D \cup\{\infty\})$ be the solution of problem $\left(P_{v_{k}}\right)$ given by (3.1). By Corollary 3.3, the sequence $\left(u_{k}\right)_{k}$ decreases to a function $u$, and since $\gamma<0$ the sequence $\left(u_{k}-v_{k} \varrho_{0}(x)\right)_{k}$ increases to $u$. Therefore, by using (3.1), (3.6) and the fact that $\gamma<0$, we obtain for each $x \in D$,

$$
\begin{aligned}
u(x) & \geq u_{k}(x)-v_{k} \varrho_{0}(x)=\int_{D} \Gamma(x, y) p(y) u_{k}^{\gamma}(y) d y \\
& \geq \beta_{k}^{\gamma} \int_{D} \Gamma(x, y) p(y)\left(\varrho_{0}(y)\right)^{\gamma} d y>0,
\end{aligned}
$$

where $\beta_{k}:=v_{k}+v_{k}^{\gamma}\|h\|_{\infty}$ and $h$ is given by (3.2).

By the monotone convergence theorem, we obtain

$$
u(x)=\int_{D} \Gamma(x, y) p(y) u^{\gamma}(y) d y
$$

Since for each $x \in D, u(x)=\inf _{k} u_{k}(x)=\sup _{k}\left(u_{k}(x)-v_{k} \varrho_{0}(x)\right)$, then $u$ is an upper and lower semicontinuous function on $D$ and so $u \in C(D)$.

Since the function $y \mapsto p(y) u^{\gamma}(y)$ is in $L_{\text {loc }}^{1}(D)$ and from (3.9) the function $x \mapsto \mathcal{N}\left(p u^{\gamma}\right)(x)$ is also in $L_{\mathrm{loc}}^{1}(D)$, we deduce by $(1.15)$ that

$$
-\Delta u(x)=p(x) u^{\gamma}(x), \quad x \in D, \text { (in the distributional sense) }
$$


Finally, using the fact that $u_{k}$ is a solution of problem $\left(P_{v_{k}}\right)$ and that $0<u(x) \leq u_{k}(x)$, for $x \in D$, we obtain

$$
\lim _{|x| \rightarrow 0}|x|^{n-2} u(x)=0 \quad \text { and } \quad \lim _{|x| \rightarrow \infty} u(x)=0 .
$$

Hence, $u$ is a solution of problem (1.5).

Proof of Theorem 1.4 Under assumption (H), by Proposition 2.12, there exists $M \geq 1$ such that for each $D$,

$$
\frac{1}{M} \theta(x) \leq \mathcal{N} q(x) \leq M \theta(x),
$$

where $\theta$ is the function defined in (1.10) and $q(y):=p(y) \theta^{\gamma}(y)$.

We split the proof into two cases.

Case 1: $\gamma<0$.

By Proposition 3.4 problem (1.5) has a positive continuous solution $u$ satisfying (3.9). We claim that $u$ satisfies (1.9).

By (3.10), we have

$$
M^{\gamma}(\mathcal{N} q)^{\gamma}(x) \leq \theta^{\gamma}(x) \leq M^{-\gamma}(\mathcal{N} q)^{\gamma}(x)
$$

Let $m=M^{-\frac{\gamma}{1-\gamma}}$. Then, by elementary calculus we have

$$
m \mathcal{N} q=\mathcal{N}\left(p(m \mathcal{N} q)^{\gamma}\right)+\mathcal{N} f
$$

where $f(x):=m p(x)\left[\theta^{\gamma}(x)-M^{\gamma}(\mathcal{N} q)^{\gamma}(x)\right]$, for $x \in D$.

Clearly, we have $f \in \mathcal{B}^{+}(D)$ and by using (3.9) and (3.12), we obtain

$$
m \mathcal{N} q-u+\mathcal{N}\left(p\left(u^{\gamma}-(m \mathcal{N} q)^{\gamma}\right)\right)=\mathcal{N} f .
$$

Let $g$ be the function defined on $D$ by

$$
g(x)= \begin{cases}p(x) \frac{u^{\gamma}(x)-(m \mathcal{N} q)^{\gamma}(x)}{(m \mathcal{N} q)(x)-u(x)}, & \text { if } u(x) \neq(m \mathcal{N} q)(x), \\ 0, & \text { if } u(x)=(m \mathcal{N} q)(x) .\end{cases}
$$

Since $\gamma<0$, then $g \in \mathcal{B}^{+}(D)$ and we have

$$
p\left(u^{\gamma}-(m \mathcal{N} q)^{\gamma}\right)=g(m \mathcal{N} q-u) .
$$

Therefore, the relation (3.13) becomes

$$
m \mathcal{N} q-u+\mathcal{N}(g(m \mathcal{N} q-u))=\mathcal{N} f
$$

Now, since $f \in \mathcal{B}^{+}(D)$ by using (3.14), (3.9), (3.12) and (3.10), we obtain

$$
\mathcal{N}(g|m \mathcal{N} q-u|) \leq \mathcal{N}\left(p u^{\gamma}\right)+\mathcal{N}\left(p(m \mathcal{N} q)^{\gamma}\right)
$$




$$
\begin{aligned}
& \leq u+m \mathcal{N} q \\
& \leq u+m M \theta<\infty .
\end{aligned}
$$

Hence, by Lemma 3.2, we obtain

$$
u \leq m \mathcal{N} q
$$

Similarly, we prove that

$$
\frac{1}{m} \mathcal{N} q \leq u
$$

Thus, by (3.10) $u$ satisfies (1.9).

Case 2: $0 \leq \gamma<1$.

Let $\omega(x)=\frac{1}{\varrho_{0}(x)} \theta(x)$, for $x \in D$. By (3.10), we have

$$
\frac{1}{M} \omega(x) \leq \frac{1}{\varrho_{0}(x)} \mathcal{N} q(x) \leq M \omega(x)
$$

Put $c=M^{\frac{1}{1-\gamma}}$ and consider the closed convex set given by

$$
E=\left\{v \in C_{0}\left(\mathbb{R}^{n}\right), \frac{1}{c} \omega \leq v \leq c \omega\right\}
$$

Note that $\omega \in E$. So $E \neq \phi$.

Define the operator $\mathbb{T}$ on $E$ by

$$
\mathbb{T} v(x):=\frac{1}{\varrho_{0}(x)} \int_{D} \Gamma(x, y) p(y)\left(\varrho_{0}(y)\right)^{\gamma} v^{\gamma}(y) d y, \quad x \in D
$$

By using (3.15), we obtain for all $v \in E$,

$$
\frac{1}{c} \omega \leq \mathbb{T} v \leq c \omega
$$

For all $v \in E$, we have

$$
\left|v^{\gamma}(y)\right| \leq c^{\gamma}\left\|\omega^{\gamma}\right\|_{\infty}, \quad \text { for all } y \in D
$$

Therefore, as in the proof of Proposition 2.6, we deduce that

$$
\mathbb{T} v \in C_{0}\left(\mathbb{R}^{n}\right), \quad \text { for all } v \in E
$$

Hence, $\mathbb{T}(E) \subset E$.

Let $\left(\omega_{k}\right)_{k} \subset C_{0}\left(\mathbb{R}^{n}\right)$ be defined by

$$
\omega_{0}=\frac{1}{c} \omega \quad \text { and } \quad \omega_{k+1}=\mathbb{T} \omega_{k}, \quad \text { for } k \in \mathbb{N} \text {. }
$$


Since the operator $\mathbb{T}$ is nondecreasing and $\mathbb{T}(E) \subset E$, we obtain

$$
\frac{1}{c} \omega=\omega_{0} \leq \omega_{1} \leq \omega_{2} \leq \cdots \leq \omega_{k} \leq \omega_{k+1} \leq c \omega .
$$

So, by the convergence monotone theorem, the sequence $\left(\omega_{k}\right)_{k}$ converges to a function $v$ satisfying for each $x \in D$,

$$
\frac{1}{c} \omega(x) \leq v(x) \leq c \omega(x) \quad \text { and } \quad v(x)=\frac{1}{\varrho_{0}(x)} \int_{D} \Gamma(x, y) p(y)\left(\varrho_{0}(y)\right)^{\gamma} v^{\gamma}(y) d y .
$$

Since $v$ is bounded, we prove by similar arguments as in the proof of Proposition 2.6 that $v \in C_{0}\left(\mathbb{R}^{n}\right)$.

Put $u(x)=\varrho_{0}(x) v(x)$. Then, $u \in C(D)$ and satisfies the equation

$$
u(x)=\mathcal{N}\left(p u^{\gamma}\right)(x), \quad \text { for } x \in D .
$$

Finally, since the function $y \mapsto p(y) u^{\gamma}(y)$ is in $L_{\text {loc }}^{1}(D)$ and from (3.16) the function $x \mapsto$ $\mathcal{N}\left(p u^{\gamma}\right)(x)$ is also in $L_{\text {loc }}^{1}(D)$, we deduce by (1.15) that $u$ is a solution of problem (1.5). The proof of Theorem 1.4 is completed.

Example 3.5 Let $\gamma<1$ and $p \in C(D)$, such that

$$
p(x) \approx|x|^{-\mu}\left(\log \left(\frac{3}{|x| \wedge 1}\right)\right)^{-\beta}(|x|+1)^{\mu-2}(\log (3|x| \vee 3))^{-2},
$$

where $\mu<n+(2-n) \gamma$ and $\beta \in \mathbb{R}$. Then, by Theorem 1.4, problem (1.5) has at least one positive solution $u \in C(D)$ satisfying the following estimates:

(i) If $2<\mu<n+(2-n) \gamma$, then for $x \in D$,

$$
u(x) \approx|x|^{\frac{2-\mu}{1-\gamma}}\left(\log \left(\frac{3}{|x| \wedge 1}\right)\right)^{\frac{-\beta}{1-\gamma}}(\log (3|x| \vee 3))^{\frac{-1}{1-\gamma}} .
$$

In particular, $\lim _{|x| \rightarrow 0} u(x)=\infty$.

(ii) If $\mu=2$ and $\beta>1$ or $\mu<2$, then for $x \in D$,

$$
u(x) \approx(\log (3|x| \vee 3))^{\frac{-1}{1-\gamma}} .
$$

(iii) If $\mu=2$ and $\beta=1$, then for $x \in D$,

$$
u(x) \approx\left(\log _{2}\left(\frac{3}{|x| \wedge 1}\right)\right)^{\frac{1}{1-\gamma}}(\log (3|x| \vee 3))^{\frac{-1}{1-\gamma}}
$$

(iv) If $\mu=2$ and $\beta<1$, then for $x \in D$,

$$
u(x) \approx\left(\log \left(\frac{3}{|x| \wedge 1}\right)\right)^{\frac{1-\beta}{1-\gamma}}(\log (3|x| \vee 3))^{\frac{-1}{1-\gamma}}
$$




\section{Funding}

The first author would like to extend his sincere appreciation to the Deanship of Scientific Research at King Saud University for its funding of this Research group NO (RG-1435-043).

Availability of data and materials

Not applicable.

\section{Declarations}

Competing interests

The authors declare that they have no competing interests.

Authors' contributions

All authors contributed equally to the writing of this paper. All authors read and approved the final manuscript.

\section{Author details}

${ }^{1}$ College of Science, Mathematics Department, King Saud University, P.O. Box 2455, 11451 Riyadh, Saudi Arabia.

${ }^{2}$ Mathematics Department, College of Science, Jouf University, P.O. Box 2014, 72338 Sakaka, Saudi Arabia.

\section{Publisher's Note}

Springer Nature remains neutral with regard to jurisdictional claims in published maps and institutional affiliations.

Received: 24 November 2021 Accepted: 3 January 2022 Published online: 14 January 2022

\section{References}

1. Bachar, I., Mâagli, H., Rădulescu, V.D.: Singular solutions of a nonlinear elliptic equation in a punctured domain. Electron. J. Qual. Theory Differ. Equ. 2017, 94 (2017)

2. Bingham, N.H., Goldie, C.M., Teugels, J.L.: Regular Variation. Encyclopedia Math. Appl., vol. 27. Cambridge University Press, Cambridge (1989)

3. Bliedtner, J., Hansen, W.: Potential Theory. An Analytic and Probabilistic Approach to Balayage. Springer, Berlin (1986)

4. Brezis, H., Kamin, S.: Sublinear elliptic equations in $\mathbb{R}^{n}$. Manuscr. Math. 74, 87-106 (1992)

5. Chemmam, R., Dhifli, A., Mâagli, H.: Asymptotic behavior of ground state solutions for sub-linear and singular nonlinear Dirichlet problem. Electron. J. Differ. Equ. 2011, 88 (2011)

6. Chung, K.L., Zhao, Z.: From Brownian Motion to Schrödinger's Equation. Springer, Berlin (1995)

7. Cîrstea, F., Rădulescu, V.D.: Existence and uniqueness of positive solutions to a semilinear elliptic problem in $\mathbb{R}^{N}$. J. Math. Anal. Appl. 229, 417-425 (1999)

8. Cîrstea, F., Rădulescu, V.D.: Uniqueness of the blow-up boundary solution of logistic equations with absorption. C. R. Math. Acad. Sci. Paris 335(5), 447-452 (2002)

9. Corduneanu, C.: Integral Equations and Stability of Feedback Systems. Academic Press, New York (1973)

10. Dalmasso, R.: Solutions d'équations elliptiques semi-linéaires singulières. Ann. Mat. Pura Appl. 153, 191-201 (1988)

11. Ghergu, M., Radulescu, V.D.: Ground state solutions for the singular Lane-Emden-Fowler equation with sublinear convection term. J. Math. Anal. Appl. 333, 265-273 (2007)

12. Ghergu, M., Rădulescu, V.D.: Singular Elliptic Problems. Bifurcation and Asymptotic Analysis. Oxford Lecture Series in Mathematics and Applications, vol. 37. Oxford University Press, London (2008)

13. Ghergu, M., Rădulescu, V.D.: PDEs Mathematical Models in Biology, Chemistry and Population Genetics. Springer Monographs in Mathematics. Springer, Heidelberg (2012)

14. Karamata, J.: Sur un mode de croissance régulière. Théorèmes fondamentaux. Bull. Soc. Math. Fr. 61, 55-62 (1933)

15. Kusano, T., Manojlović, J.: Asymptotic behavior of positive solutions of sublinear differential equations of Emden-Fowler type. Comput. Math. Appl. 62(2), 551-565 (2011)

16. Kusano, T., Manojlović, J.: Positive solutions of fourth order Thomas-Fermi type differential equations in the framework of regular variation. Acta Appl. Math. 121, 81-103 (2012)

17. Kusano, T., Manojlović, J.: Positive solutions of fourth order Emden-Fowler type differential equations in the framework of regular variation. Appl. Math. Comput. 218(12), 6684-6701 (2012)

18. Lair, A.V., Shaker, A.W.: Entire solution of a singular semilinear elliptic problem. J. Math. Anal. Appl. 200, 498-505 (1996)

19. Mâagli, H.: Asymptotic behavior of positive solutions of a semilinear Dirichlet problem. Nonlinear Anal. 74, 2941-2947 (2011)

20. Mâagli, H., Zribi, M.: Existence and estimates of solutions for singular nonlinear elliptic problems. J. Math. Anal. Appl. 263(2), 522-542 (2001)

21. Maric, V.: Regular Variation and Differential Equations. Lecture Notes in Math., vol. 1726. Springer, Berlin (2000)

22. Mi, L.: Existence and boundary behavior of solutions to p-Laplacian elliptic equations. Bound. Value Probl. 2016, 119 (2016)

23. Port, S.C., Stone, C.J.: Brownian Motion and Classical Potential Theory. Academic Press, San Diego (1978)

24. Řehák, P.: Nonlinear differential equations in the framework of regular variation (2014)

25. Seneta, R.: Regularly Varying Functions. Lectures Notes in Math., vol. 508. Springer, Berlin (1976)

26. Shaker, A.W.: On singular semilinear elliptic equations. J. Math. Anal. Appl. 173, 222-228 (1993)

27. Zhao, Z:: Green function for Schrödinger operator and conditional Feynman-Kac gauge. J. Math. Anal. Appl. 116 309-334 (1986)

28. Zhao, Z:: On the existence of positive solutions of nonlinear elliptic equations. A probabilistic potential theory approach. Duke Math. J. 69, 247-258 (1993) 